\title{
Further Investigation on the High-Conductance Ion Channel of the Inner Membrane of Mitochondria
}

\author{
M. Catia Sorgato, ${ }^{1}$ Oscar Moran, ${ }^{2}$ Vito De Pinto, ${ }^{3}$ Bernhard U. Keller, ${ }^{4}$ \\ and Walter Stuehmer ${ }^{4}$
}

Received June L, 1989

\begin{abstract}
By use of the patch-clamp technique, the inner membrane of mouse liver and heart mitochondria is shown to contain a highly conductive (around $100 \mathrm{pS}$ in symmetrical $150 \mathrm{mM} \mathrm{KCl}$ ) and voltage-dependent ion channel. This channel closely resembles that previously found in cuprizone-treated mouse liver inner mitochondrial membrane. The paper discusses the electrical properties of the channel and its possible physiological function. The reconstitution in giant liposomes of a partially purified ox heart inner membrane fraction containing the channel and the use of various inhibitors are also presented.
\end{abstract}

Key Words: Patch clamp; planar membranes; liver mitochondria; heart mitochondria; liposomes; ion channel; IMM channel (inner mitochondrial membrane channel).

\section{Introduction}

From its original application to cell membranes (Hamill et al., 1981), the patch-clamp technique has recently been successfully extended to subcellular membranes. For instance, it has been possible to detect, at the molecular level, highly conductive ion channels in vacuoles (Hedrich et al., 1986), in the thylakoid membrane of plant cells (Schoenknecht et al., 1988), and in the sarcoplasmic reticulum of frog muscle fibers (Stein and Palade, 1988). By enlarging purified membranes, in order to obtain vesicles large enough to be patch clamped, ion channels have also been found in synaptic vesicles (Rahamimoff et al., 1988) and in endoplasmic reticulum (Keller et al., 1988a).

\footnotetext{
'Dipartimento di Chimica Biologica e Centro CNR per lo Studio della Fisiologia Mitocondriale, Universitá di Padova, Padova, Italy.

${ }^{2}$ Lab. di Biofisica, Scuola Internazionale Superiore di Studi Avanzati, Trieste, Italy.

${ }^{3}$ Dipartimento Farmaco-Biologico, Universitá di Bari, Bari, Italy.

${ }^{4}$ Max Planck Institut fur biophysikalische Chemie, Abt. Membranbiophysik, Goettingen, FRG.
} 
We have applied the patch clamp to liver mitoplasts, vesicles made of the inner mitochondrial membrane (Sorgato et al., 1987). It has been possible to record single channels with high conductance properties which are exquisitely voltage gated, in the sense that they open only upon depolarization of the membrane potential which is physiologically maintained across the inner membrane in respiring mitochondria (Ferguson and Sorgato, 1982). At first sight, these characteristics of the inner mitochondrial membrane (IMM $)$ channel do not fit easily into the context of our current knowledge of mitochondrial ion transport (LaNoue and Schoolwerth, 1979; see also Beavis, 1989), and of the bioenergetic rules applicable to mitochondria. For this reason we have considered various other possibilities for the role of the channel. Additionally, since the patched mitoplasts were obtained from liver mitochondria of mice fed with cuprizone, we had to eliminate the possibility that the channel could somehow be the consequence of the special diet. Indeed, thus drug induces the formation of abnormally large liver mitochondria, with some biochemical alterations (Tandler and Hoppel, 1973; Hoppel and Tandler, 1973).

This paper demonstrates that, by testing various blockers or modifiers of known channels and of some mitochondrial proteins, it is possible to discriminate among some of the hypothetical roles of the IMM channel. It also shows that a highly conductive ion channel is found in mitoplasts derived from liver and heart of animals not treated with cuprizone. Finally, it is reported that the channel is present in large liposomes containing partially purified fractions of the inner membrane of ox heart mitochondria and consequently that this method of constitution can be successfully used to trace the channel during its purification.

\section{Materials and Methods}

\section{Chemicals}

ACA 34 was purchased from Pharmacia. All other reagents were from Sigma. Ruthenium red was purified according to Luft (1971).

\section{Preparation of Mitochondria and Mitoplasts}

Giant mitochondria were obtained from the liver of mice maintained on a cuprizone-enriched diet for at least 4 days (Bowman and Tedeschi, 1983).

\footnotetext{
${ }^{5}$ Abbreviations: IMM, inner mitochondrial membrane; DCCD, $N, N^{\prime}$-dicyclohexylcarbodiimide; DIDS, 4-4'-diisothiocyanatostilbene-2,2'-disulfonic acid; cuprizone, biscyclohexanone oxalyldihydrazone; LDAO, lauryl dimethyl aminooxide; SDS-PAGE, sodium dodecyl sulfatepolyacrylamide-gel electrophoresis.
} 
Giant mitochondria were isolated as in Sorgato et al. (1987) and mitoplasts were obtained by swelling and shrinking the mitochondria (Sottocasa et al., 1967).

The same procedures were followed to prepare mitochondria and mitoplasts from liver and heart of cuprizone-untreated mice. With these treatments it was possible to obtain mitoplasts large enough to be visible with the optical microscope (using a magnification of $800-1600$-fold). Frequently a second swelling, for approximately $10 \mathrm{~min}$, was performed on the dish for further enlargement of the vesicles. The preparation was then extensively perfused with the solution to be used for the electrical recordings. For preparing heart mitochondria, originally the heart tissue was treated with collagenase (Toth et al., 1986). The use of collagenase was, however, quickly abandoned because it rendered the membranes of the vesicles soft and fragile and thus difficult to patch.

\section{Preparation of Inner Membrane Fractions from Ox Heart Mitochondria}

The inner mitochondrial membrane fraction rich in ATPase activity was prepared according to Stiggall et al. (1979). This was treated with LDAO (2\% final concentration) and then loaded on a $100 \times 1 \mathrm{~cm}$ column of ultragel ACA 34 equilibrated with $0.1 \% \mathrm{LDAO}, 10 \mathrm{mM}$ Tris $\mathrm{HCl}(\mathrm{pH} 7)$, and $1 \mathrm{mM}$ EDTA. The column was eluted with the equilibration buffer and the resulting fractions centrifuged for $45 \mathrm{~min}$ at maximal speed in an Airfuge. The pellets were then mixed with liposomes (Keller et al., 1988b) and the liposomes enlarged as described below.

\section{Preparation of $F_{0}$ and of $F_{0}$-Containing Liposomes}

$\mathrm{F}_{0}$ was extracted with urea (Galante et al., 1981) from ox heart ATP synthase isolated according to Stiggall et al. (1979). $\mathrm{F}_{0}$ was then inserted in asolectin liposomes according to Lippe et al. (1988), except that the $\mathrm{pH}$ of the resulting proteoliposomes was always brought to 7.0.

\section{Preparation of Giant Liposomes}

Enlargement of protein-containing liposomes, suitable for patch clamping, was accomplished by careful dehydration and subsequent rehydration of the proteoliposomes, as described by Criado and Keller (1987).

\section{Electrical Recording}

Patch-Clamp Experiments. The patch-clamp setup used in the experiments was as described by Hamill et al. (1981). Both vesicles and liposomes were prepared for the experiments as detailed in Sorgato et al. (1987). Bath 
and pipette solution was usually $150 \mathrm{mM} \mathrm{KCl}$ and $20 \mathrm{mM}$ Hepes-KOH (pH 7.2), except for the experiments with giant liposomes in which $5 \mathrm{mM}$ Hepes was used. If present, $\mathrm{CaCl}_{2}$ was $0.1 \mathrm{mM}$. Pipettes with $5-30 \mathrm{M} \Omega$ resistance was used. Seals were greater than $10 \mathrm{G} \Omega$ resistance. Current records were stored in a RACAL 4DSFM tape recorder, filtered at $1 \mathrm{kHz}$ (8-pole Bessel filter), and stored in a computer for analysis and graphical display. Single-channel current records were analyzed with the computer program TAC (Sigworth, 1983). Temperature was room temperature.

Unless otherwise specified, the given sign of the potential for the cell (mitoplast)-attached configuration (Hamill et al., 1981) is, following the physiological convention, that found inside the vesicle.

Planar Lipid Bilayer Recording. Black lipid membranes were formed as described by Mueller and Rudin (1969) and detailed in Stuehmer et al. (1988). Liposomes containing 150-200 $\mu \mathrm{g}$ ox heart $\mathrm{F}_{0}$ were added to the cis chamber and fused with the planar membrane according to Miller and Racker (1976). The solutions in the chambers contained $0.25 \mathrm{mM}$ dithiothreitol, $1.8 \mathrm{mM}$ $\mathrm{CaCl}_{2}, 2.5 \mathrm{mM} \mathrm{MgSO}_{4}, 0.2 \mathrm{mM}$ EDTA, $100 \mathrm{mM} \mathrm{NaCl}$, and $10 \mathrm{mM}$ Tricine$\mathrm{NaOH}$. For electrical measurements, a List Medical Electronics EPC-7 amplifier was used in voltage-clamp mode. $\mathrm{Ag} / \mathrm{AgCl}$ pellet electrodes were used, and the cis electrode was set to a command voltage relative to the trans electrode, held at virtual ground. Current output was filtered at $3 \mathrm{kHz}$ and recorded on a RACAL 4DSFM tape recorder for further analysis. Temperature was room temperature.

\section{Results}

\section{Patch Clamping of Mitoplasts}

From Cuprizone-Treated Mouse Liver. The first attempt to patch clamp the inner membrane of mitochondria (Sorgato et al., 1987) was performed on mitoplasts obtained from the liver of mice maintained on a diet rich in cuprizone. This compound is known to induce the formation of larger liver mitochondria (Tandler and Hoppel, 1973), which, after outer membrane removal by osmotic shock (Sottocasa et al., 1967), yield vesicles (mitoplasts) which can be fairly easily patch clamped. The electrical recording shows the presence of an ion channel with approximately $100 \mathrm{pS}$ conductance (in symmetrical $150 \mathrm{~mm} \mathrm{KCl}$ ) whose opening is voltage dependent (Fig. 1). At potential values more negative than approximately $-50 \mathrm{mV}$, this channel was always in the closed state (Sorgato et al., 1987).

From Cuprizone-Untreated Mouse Liver. The 2-3- $\mu$ m-diameter mitochondria obtained by us from cuprizone-treated mice (Sorgato et al., 1987) were not as big as previously reported in the literature (Tandler and Hoppel, 


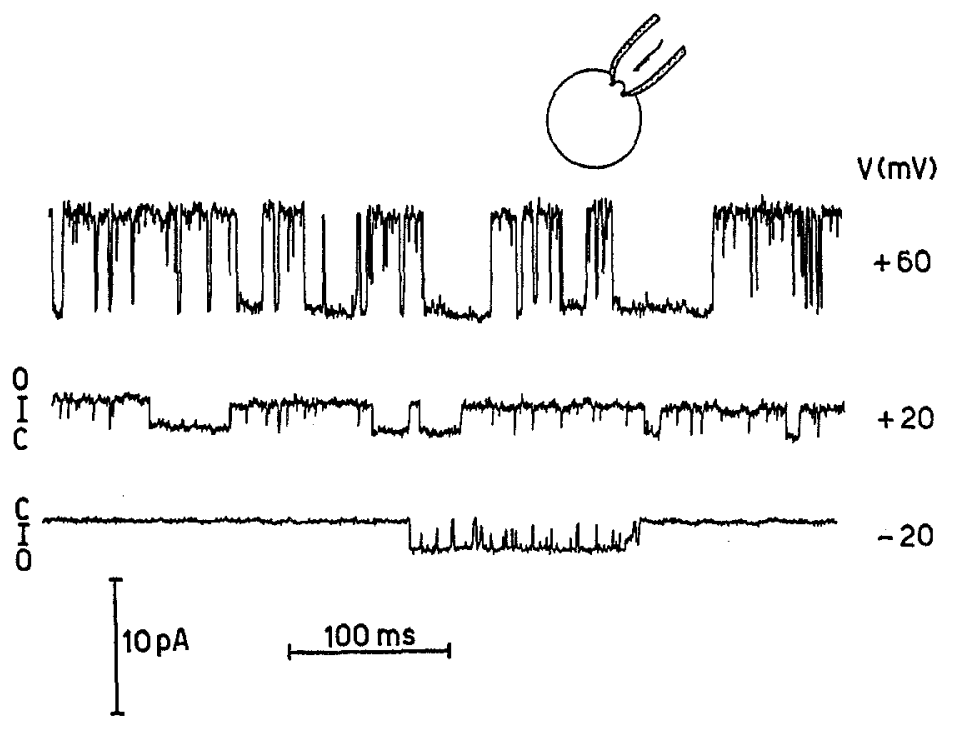

Fig. 1. Current records at different potentials of a liver mitoplast obtained from mice treated with cuprizone. The vesicle was in the cell-attached configuration and the solutions were symmetrical $150 \mathrm{mM} \mathrm{KCl}$ (see Materials and Methods). The sign of the potential shown refers to that inside of the mitoplast.

1973; Bowman and Tedeschi, 1983). This finding prompted us to try to isolate the larger mitochondria from cuprizone-untreated liver, and then to obtain mitoplasts, by following the same procedures used to prepare cuprizone mitochondria and mitoplasts. The size of the mitoplasts from cuprizoneuntreated liver was on average smaller than that of mitoplasts from cuprizonetreated liver. But it could be augmented by a second incubation with the swelling buffer, which was usually performed directly in the patch-clamp setup (see Materials and Methods). This double swelling eventually yielded vesicles with a maximum diameter of approximately $3 \mu \mathrm{m}$, amenable to patchclamp investigation. Figure 2 shows that in the inner membrane of normal liver mitochondria a channel can be found with the same conductance and voltage dependence as that of liver mitoplasts from cuprizone-treated animals.

From Mouse Heart. The same procedures outlined for preparing normal liver mitoplasts were adopted to prepare heart mitoplasts. Also in these membranes a channel was seen with the same electrical characteristics as those shown in Fig. 2 (data not shown).

\section{Patch-Clamping of Liposomes}

In a previous paper (Stuehmer et al., 1988) we reported that a highconductance channel, with similar characteristics to the IMM channel detected 


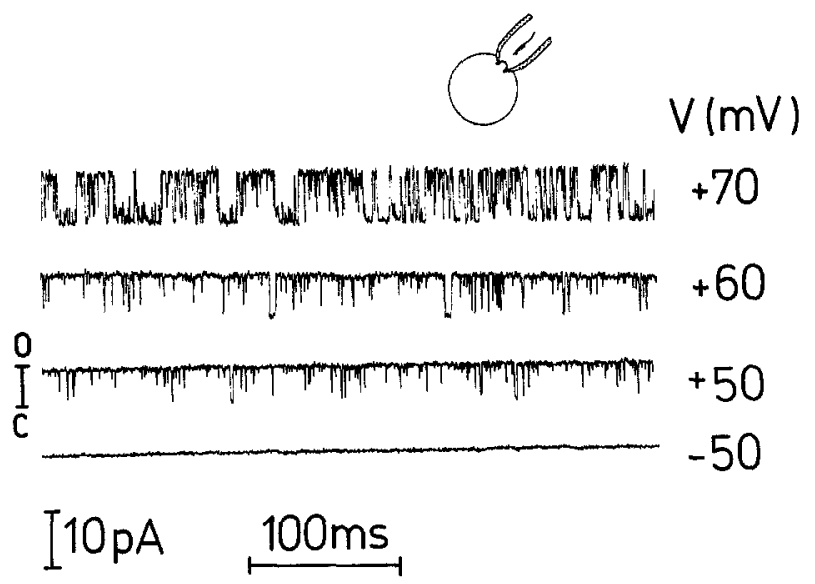

Fig. 2. Current records at different potentials of a liver mitoplast obtained from mice not treated with cuprizone. The vesicle was in the cell-attached configuration. The solutions used and the meaning of the sign of the potential are as in Fig. 1.

in mouse liver, was found when $F_{0}$ (the membrane part of ATP synthase) was incorporated in planar membranes. We concluded that the IMM channel was coprecipitating with the enzyme during its isolation. This was likely since gel electrophoresis patterns of the ATP synthase (and also of the $F_{0}$ extracted from it) contained a number of unknown bands (Lippe et al., 1988). Therefore, as a first step to isolate the IMM channel, the ATP synthase complex was isolated from ox heart mitochondria and chromatographed on an ultragel ACA-34 column. This resulted in fractions which had fewer bands in SDS-PAGE below $20 \mathrm{kDa}$ than the starting material (data not shown). The fractions were then incorporated in liposomes and subsequently enlarged by a dehydration-rehydration step (see Materials and Methods).

The patch clamping of these liposomes reveals a channel with the same conductance $(95 \mathrm{pS})$ and voltage dependence as the IMM channel described above (Fig. 3). The data confirm the results obtained with planar membranes (Stuehmer et al., 1988) and show that the IMM channel is present in heart mitochondria of different animals (mouse and ox). Most importantly, this result also shows that incorporation of the channel in large liposomes does not alter its electrical properties and, consequently, that this method can be used to monitor the presence of the channel during its isolation.

\section{Test with Inhibitors}

Patch-Clamp Experiments of Cuprizone-Free Mitoplasts. A number of compounds have been used in order to find an inhibitor of the IMM channel and also, by comparison, to identify the role of the channel (Table I, column A). 


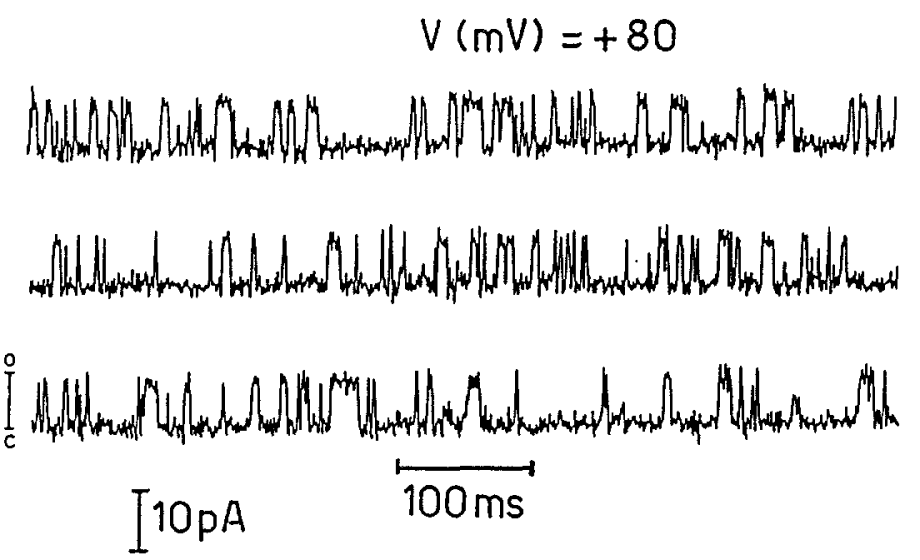

Fig. 3. Current record of liposomes containing an inner mitochondrial membrane fraction. The patch was excised. Preparation of the mitochondrial fraction and of giant proteoliposomes is as detailed in Materials and Methods. Experimental conditions for the recording are as in Fig. I.

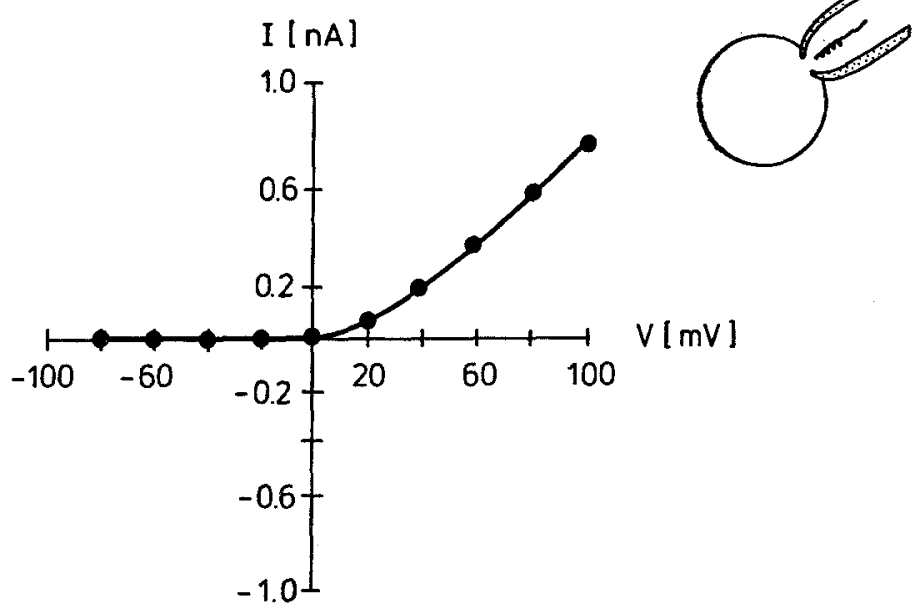

Fig. 4. Current-voltage relation of a cuprizone-free mitoplast in the whole-cell configuration, under steady-state conditions. Solutions used were symmetrical $150 \mathrm{mM} \mathrm{KCl}$ (see Materials and Methods).

None had an effect on the electrical activity of the channel. The reagents were either added to both the bath and the pipette solution or only to the bath. Since we preferentially used mitoplasts in the whole-cell configuration for these tests, the possible effect of the added compounds was followed in terms of the typical asymmetric current-voltage trace obtained with this configuration (Fig. 4) (see also Sorgato et al., 1987). 
Table I. List of Reagents Used in Patch-Clamp and Planar Membrane Experiments ${ }^{a}$

\begin{tabular}{|c|c|c|}
\hline \multirow{2}{*}{$\begin{array}{c}\text { Reagents } \\
\text { (concentrations) }\end{array}$} & $\mathrm{A}$ & B \\
\hline & Patch clamp & Planar membrane \\
\hline $\begin{array}{l}\text { Oligomycin } \\
(0.015 \mathrm{mM})\end{array}$ & - & - \\
\hline $\begin{array}{l}\text { DCCD } \\
(0.011 \mathrm{mM})\end{array}$ & - & - \\
\hline $\begin{array}{l}\text { Bongkrekic acid } \\
(0.250 \mathrm{mM})\end{array}$ & - & - \\
\hline $\begin{array}{l}\text { DIDS } \\
(0.15-1 \mathrm{mM})\end{array}$ & - & $\mathrm{n} / \mathrm{t}$ \\
\hline $\begin{array}{l}\mathrm{ZnCl}_{2} \\
(0.1 \mathrm{mM})\end{array}$ & - & $\mathrm{n} / \mathrm{t}$ \\
\hline $\begin{array}{l}\text { Quinine } \\
(0.2 \mathrm{mM})\end{array}$ & - & $\mathrm{n} / \mathrm{t}$ \\
\hline $\begin{array}{l}\mathrm{GdCl}_{3} \\
(0.01-0.03 \mathrm{mM})\end{array}$ & - & $\mathrm{n} / \mathrm{t}$ \\
\hline $\begin{array}{l}\text { Decamethonium } \\
(1-5 \mathrm{mM})\end{array}$ & - & $\mathrm{n} / \mathrm{t}$ \\
\hline $\begin{array}{l}\text { Carboxyatractylate } \\
(0.005-0.05 \mathrm{mM})\end{array}$ & $\mathrm{n} / \mathrm{t}$ & - \\
\hline $\begin{array}{l}\text { Ruthenium red } \\
(0.001 \mathrm{mM})\end{array}$ & $\mathrm{n} / \mathrm{t}$ & - \\
\hline $\begin{array}{l}\text { Tetrodotoxin } \\
(0.01 \mathrm{mM})\end{array}$ & $\mathrm{n} / \mathrm{t}$ & - \\
\hline $\begin{array}{l}\text { Tetraethylammonium } \\
(1-5 \mathrm{mM})\end{array}$ & $\mathrm{n} / \mathrm{t}$ & - \\
\hline
\end{tabular}

"In patch-clamp experiments, the effect of the added compounds on current-voltage relations in the whole-cell configuration was tested (Fig. 4). In planar membrane experiments, effects on single-channel currents were followed (Stuehmer et al, 1988). A dash indicates that the compound was tested and had no detectable effect on the IMM channel. " $n / t$ " indicates that the compound was not tested under a particular condition. The final concentration or the range of final conccentrations used are given in parentheses. Oligomycin and DCCD were dissolved in ethanol, and bongkrekic acid in $\mathrm{NH}_{4} \mathrm{OH}$. The final concentration of these solvents was maximally $1 \%(\mathrm{v} / \mathrm{v})$. In the patch-clamp experiments, all reagents listed were added in both solutions, except for bongkrekic acid present only in the bath solution. Cuprizone-free mitoplasts were used, except when decamethonium was tested, in which case cuprizone mitoplasts were patched. For other experimental conditions see Materials and Methods.

In Planar Membranes. The reagents listed in Table I, column B, were either added to the cis chamber after $\mathrm{F}_{0}$-containing liposomes had been incorporated into planar membranes (see Materials and Methods) or, in some cases, were incubated with $\mathrm{F}_{0}$ before its addition to the chamber. Under none of these experimental conditions did the reagents have an effect on the activity of the single channel which could be recorded with this system (Stuehmer et al., 1988). 


\section{Discussion}

Since the first finding of a high-conductance ion channel in the inner membrane of mitochondria by use of an electrophysiological technique (Sorgato et al., 1987), other electrophysiological studies of mitochondria have confirmed the existence of such entities (Thieffry et al., 1988; Kinnally et al., 1988a, 1988b). It seems therefore established that, contrary to some (but not a11) expectations, these channels must play a role in the physiology of mitochondria. Unfortunately, no one is yet in the position to assign a definite role to the channel(s). Nonetheless, we at least can discriminate among some of the possibilities.

On the basis of data obtained with nonelectrophysiological methods, the existence of a high-conductance anion uniporter in the inner membrane of mitochondria has already been postulated by some authors (for reviews, see Garlid and Beavis, 1986; Beavis, 1989). It has been hypothesized that this channel would function under abnormal conditions, namely a high matrix $\mathrm{pH}$ or depletion of divalent cations at physiological $\mathrm{pH}$. The possibility that the IMM channel is this anion channel must therefore be considered. Although its selectivity is far from absolute, the IMM channel seems also to transport preferentially anions (Sorgato et al., 1987). However, unlike the anion uniporter, an alkaline $\mathrm{pH}$ was found not to function as a physiological modulator of the IMM channel (Sorgato et al., 1987). The lack of inhibition by quinine and DCCD [blockers of the anion uniporter (Beavis and Garlid, 1988; Beavis, 1989)], shown in Table I, further militates against the identification of the anion uniporter with the IMM channel described by us.

Oligomycin and DCCD, two well-known inhibitors of the $F_{0}$ part of the ATP synthase, were used in both planar membrane and mitoplast patchclamping experiments (Table I). The lack of effect of these reagents in either condition argues against the possibility that the IMM channel might be $F_{0}$ itself. A single channel, with characteristics similar to those detected by patch-clamping mitoplasts, was recorded with ox heart $F_{0}$ incorporated in lipid bilayers (Stuehmer et al., 1988). We have originally interpreted these results as being due to the presence of the IMM channel in fractions of $F_{0}$ (and of the ATP synthase; see Fig. 3). We noted that the voltage dependence of the channel seen in planar membranes (and later in mitoplasts) was opposite that expected for a channel in $F_{0}$. (The IMM channel closes at potential values more negative than $-50 \mathrm{mV}$, conditions at which $\mathrm{F}_{0}$ is expected to be open to allow synthesis of ATP.) The lack of action of oligomycin and DCCD confirms this interpretation. This conclusion is important also in light of recent reports that some $\mathrm{F}_{0}$ molecules of the chloroplast ATP synthase could have a much higher conductance (in the pS 
range) than that calculated from the proton current through $\mathrm{F}_{0}$ inserted into liposomes (Lill et al., 1987; Lill and Junge, 1989).

Carboxyatractylate and bongkrekic acid were tested to ascertain whether the IMM channel is related to the adenine nucleotide translocator. In particular Andreyev et al. (1988) have postulated that the mitochondrial adenine nucleotide translocator would, in the presence of low concentrations of fatty acids, become a heat generator in muscle as well as in liver, without losing its sensitivity to the classical inhibitors. It is evident from the results of Table I that the IMM channel does not respond to these molecules and consequently that it is probably not the modified adenine nucleotide translocator involved in nonshivering thermogenesis.

No inhibition was seen with various neuronal channel blockers (decamethonium, tetrodotoxin, and tetraethylammonium) nor with modifiers of some chloride channels (DIDS and $\mathrm{ZnCl}_{2}$ ) (Table I).

Since $0.1 \mathrm{mM} \mathrm{CaCl}_{2}$ was routinely present in the patch-clamp solutions, some attention had to be given to the possibility that this unphysiologically high concentration of calcium could be responsible for the opening of unspecific pores in the inner membrane of mitochondria (Pfeiffer et al., 1978; Haworth et al., 1980; Crompton et al., 1987). This was found not to be the case because omission of calcium from both the pipette and bath solution did not result in the disappearance of the channel (Sorgato et al., 1987). Similarly, the addition of up to $100 \mu \mathrm{M}$ EGTA in calcium-free media had no effect on the detection of the channel (data not shown).

Finally, we considered the possibility that the IMM channel might be a stretch-activated channel, such as those apparently involved in the osmotic regulation of a variety of cells (Sachs, 1986; Saimi et al., 1988). Indeed they share some similarities in their conductance value and voltage dependence. However, we have observed that the extent of suction on patches effects neither the number nor open times of the IMM channel. Also gadolinium, which inhibits mammalian and plant stretch-activated channels (Yang and Sachs, 1989), has no effect on the IMM channel (Table I, column A).

We are thus left with an open question regarding the use of such a large conductance channel in the inner membrane of mitochondria. Parenthetically, this question can be placed in the more general context of the as-yet ill-defined role of ion channels recently discovered in other subcellular organelles (Hedrich et al., 1986; Keller et al., 1988a; Rahamimoff et al., 1988; Schoenknecht et al., 1988).

The possibility that the IMM channel might serve, autonomously or in conjunction with channels present in the outer membrane (Colombini, 1979; Kinnally et al., 1987), in the machinery for the import of cytoplasmically synthesized mitochondrial proteins has already been considered by us (Sorgato et al., 1987) and by others (Thieffry et al., 1988). The import apparatus 
seems to lie in those regions where the two mitochondrial membranes are juxtaposed (Hartl et al., 1989). These contact sites have also been biochemically characterized (Kottke et al., 1988). In principle, some of the features expected for pores involved in mitochondrial protein transport are displayed by the IMM channel. Work is in progress to test this possibility.

Along this line, it may also be of interest to report here that the inner membrane of mitochondria seems to have more than one highly conductive state. In fact, patch clamping of the inner membrane fraction-enriched liposomes has revealed on some occasions the presence of conductances of higher and lower values than that typical of the IMM channel (approx. $100 \mathrm{pS}$ ). While the higher conductance levels were infrequent in the native membranes of cuprizone-treated or cuprizone-untreated mitoplasts, lower conductance states were observed especially in the cuprizone-free vesicles (see Fig. 2). We are currently trying to determine whether the different currents belong to different channels or to an unique channel with different conductance states.

In summary, this paper has shown that some of the roles that could tentatively be assigned to the IMM channel are likely not to be valid. It also reports the feasibility of applying the patch-clamp technique to cuprizonefree mitoplasts of both mouse liver and heart and to liposomes containing a partially purified fraction of the inner membrane of ox heart mitochondria. Finally, the finding in all these systems of the same channel as that found in cuprizone-liver mitoplasts demonstrates that the IMM channel is not a consequence of the cuprizone diet but a physiological component of mitochondria of different tissues.

\section{Acknowledgments}

M.C.S. thanks the European Molecular Biology Organisation for a short-term fellowship. The authors gratefully thank Miss Monica Papke and Mr. Michael Pilot for their excellent help.

\section{References}

Andreyev, A. Yu., Bondareva, T. O., Dedukhova, V. I., Mokhova, E. N., Skulachev, V., and Volkov, N. I. (1988) FEBS Lett. 226, 265-269.

Beavis, A. D. (1989) J. Biol. Chem. 264, 1508-1515.

Beavis, A. D., and Garlid, K. D. (1988) J. Biol. Chem. 263, 7574-7580.

Bowman, C. L., and Tedeschi, H. (1983) Biochim. Biophys. Acta 731, 260-266.

Colombini, M. (1979) Nature (London) 279, 643-645.

Criado, M., and Keller, B. U. (1987) FEBS Lett. 224, 172-176.

Crompton, M., Costi, A., and Hayat, L. (1987) Biochem. J. 245, 915-918.

Ferguson, S. J., and Sorgato, M. C. (1982) Annu. Rev. Biochem. 51, 185-217.

Galante, Y. M., Wong, S., and Hatefi, Y. (1981) Arch. Biochem. Biophys. 211, 643-651. 
Garlid, K., and Beavis, A. D. (1986) Biochim. Biophys. Acta 853, 187-204.

Hamill, O. P., Marty, A., Neher, E., Sakmann, B., and Sigworth, F. J. (1981) Pflugers Arch. Ges. Physiol. 391, 85-100.

Hartl, F-U., Pfanner, N., Nicholson, D. W., and Neupert, W. (1989) Biochim. Biophys. Acta 988, $1-45$.

Haworth, R. A., and Hunter, D. R. (1980) J. Membr. Biol. 54, 231-236.

Hedrich, R., Fluegge, U. I., and Fernandez, J. M. (1986) FEBS Lett. 204, 228-232.

Hoppel, C. L., and Tandler, B. (1973) Biochem. Pharmacol. 22, 2311-2318.

Keller, B. U., Kleineke, J., Criado, M., and Soeling, H. D. (1988a) Pfuegers Arch. 411, Suppl. 1,105

Keller, B. U., Hedrich, R., Vaz, W. L. C., and Criado, M. (1988b) Pfuegers Arch. 411, 94-100.

Kinnally, K. W., Tedeschi, H., and Mannella, C. A. (1987) FEBS Lett. 226, 83-87.

Kinnally, K. W., Campo, M. L., and Tedeschi, H. (1988a) Abstract TH: 420 of the XIV International Congress of Biochemistry, Prague.

Kinnally, K. W., Campo, M. L., and Tedeschi, H. (1988b) J. Cell Biol. 107, 353a.

Kottke, M., Adam, V., Riesinger, I., Bremm, G., Bosch, W., Brdiczka, D., Sandri, G., and Panfili, E. (1988) Biochim. Biophys. Acta 935, 87-102.

LaNoue, K. F., and Schoolworth, A. C. (1979) Annu. Rev. Biochem. 48, 871-922.

Lill, H., and Junge, W. (1989) Eur. J. Biochem. 179, 459-467.

Lill, H., Althoff, G., and Junge, W. (1987) J. Membr. Biol. 98, 69-78.

Lippe, G., Dabbeni-Sala, F., and Sorgato, M. C. (1988) J. Biol. Chem. 263, 18627-18634.

Luft, J. M. (1971) Anat. Rec. 171, 347-368.

Miller, C., and Racker, E. (1976) J. Membr. Biol. 30, 283-300.

Mueller, P., and Rudin, D. O. (1969) In Laboratory Techniques in Membrane Biophysics (Passow, A., and Staempfli, R., eds.), Springer, Heidelberg, pp. 141-156.

Pfeiffer, D. R., Kauffman, R. F., and Lardy, H. A. (1978) J. Biol. Chem. 253, 4165-4171.

Rahamimoff, R., DeRiemer, S. A., Sakmann, B., Stadler, H., and Yakir, N. (1988) Proc. Natl. Acad. Sci. USA 85, 5310-5314.

Sachs, F. (1986) Membr. Biochem. 6, 173-195.

Saimi, Y., Martinac, B., Gustin, M. C., Culbertson, M. R., Adler, J., and Kung, C. (1988) Trends Biochem. Sci. 13, 304-309.

Schoenknecht, G., Hedrich, R., Junge, W., and Raschke, K. (1988) Nature (London) 336, 589-592.

Sigworth, F. (1983) In Single-Channel Recording (Sakmann, B., and Neher, E., eds.), Plenum Press, New York, pp. 91-105.

Sorgato, M. C., Keller, B. U., and Stuehmer, W. (1987) Nature (London) 330, 498-500.

Sottocasa, G. L., Kuylenstrierna, B., Ernster, L., and Bergstrand, A. (1967) Methods Enzymol. 10, 448-463.

Stein, P., and Palade, P. (1988) Biophys. J. 54, 357-363.

Stiggall, D. L., Galante, Y. M., and Hatef, Y. (1979) Methods Enzymol. 55, 308-315.

Stuehmer, W., Keller, B., Lippe, G., and Sorgato, M. C. (1988) In Hormones and Cell Regulation (Nunez, J., Dumont, J. E., and Carafoli, E., eds.), Colloque INSERM/John Libbey Eurotext Ltd., Vol. 165, pp. 89-99.

Tandler, B., and Hoppel, C. L. (1973) J. Biol. Chem. 56, 266-272.

Thieffry, M., Chich, J., Goldschmidt, D., and Henry, J. (1988) EMBO J. 7, 1449-1454.

Toth, P. P., Ferguson-Miller, S., and Suelter, C. H. (1986) Methods Enzymol. 125, 16-27.

Yang, X-C., and Sachs, F. (1989) Science 243, 1068-1071. 